\title{
Static and Dynamic Reliability Analysis of Laterally Loaded Pile Using Probability Density Function Method
}

\author{
Min Xiong ${ }^{1}$ and Yu Huang ${ }^{1,2, *(D)}$ \\ 1 Department of Geotechnical Engineering, College of Civil Engineering, Tongji University, \\ Shanghai 200092, China; 1310298@tongji.edu.cn \\ 2 Key Laboratory of Geotechnical and Underground Engineering of the Ministry of Education, \\ Tongji University, Shanghai 200092, China \\ * Correspondence: yhuang@tongji.edu.cn; Tel.: +86-021-6598-2384
}

Received: 17 November 2020; Accepted: 3 December 2020; Published: 5 December 2020

\begin{abstract}
Pile foundation is one of the common foundation forms in marine geotechnical engineering, especially in wind power engineering. Its operation safety is seriously affected by many uncertainties, such as the randomness of ground motion in intensity and frequency. The stochastic reliability analysis method can better characterize these uncertainties in the evaluation of the safety performance of pile foundation. The probability density functions (PDFs) of stochastic systems are important prerequisites for reliability analysis. However, for geotechnical problems, the coupling between parametric and excitation randomness and the nonlinear mechanical properties of rock and soil make it very difficult to obtain the associated PDFs. Instead, the probability density evolution method (PDEM) is introduced and is used to investigate the static and dynamic reliability of laterally loaded piles as an example of a geotechnical problem. Compared with Monte Carlo stochastic simulations, PDEM-based computing is shown to be highly efficient when applied to the seismic design of pile in geotechnical engineering, and its calculation efficiency is 20 times of the former for the seismic dynamic reliability of pile foundation. This study provides a new reference for the efficient design and safety evaluation of offshore pile foundation engineering based on static and dynamic reliability of multiple random factors.
\end{abstract}

Keywords: laterally loaded piles; reliability; probability density function; Monte Carlo simulation

\section{Introduction}

In foundation engineering involving large piles, pile foundation is one of the common structural forms of offshore wind power. It is generally the case that the axial force is the main control load and buckling is the main failure mode [1,2]. However, because of lateral action from seismic excitation, wind, waves, and various other water and soil pressures, a single pile or a pile group is usually also subjected to huge lateral loading; examples of this are offshore platforms, transmission-line towers, high-piled wharfs, and the piers of long bridges. Therefore, there have been many studies of the static and dynamic responses of piles. These range from closed-form exact solutions of simplified problems $[3,4]$ to numerical solutions of complicated nonlinear problems [5,6]. Meanwhile, many researchers have gradually come to realize the importance of uncertainty and randomness when analyzing the static and dynamic responses of a laterally loaded pile system. In pioneering work, Zhang et al. [7] systematically analyzed and summarized four main sources of analytical uncertainty and randomness for laterally loaded piles and proceeded to study the static reliability of piles considering the variability of the soil modulus. Aimed at soil-structure interaction, Barakat et al. [8] proposed a reliability and optimum analysis and design method for laterally loaded piles. 
Recently, thanks to rapid computational developments, various analyses have been conducted of the linear and nonlinear stochastic responses of laterally loaded piles using finite-element (FE) and finite-difference approaches [9-13]. These studies considered multiple correlated sources of randomness in the static reliability analysis of a laterally loaded pile using the first-order and second-order reliability methods, the response-surface method, and Monte Carlo simulation, among others. However, for Monte Carlo simulation, the anomaly of stochastic convergence means that extensive computation is required [14,15]. Obviously, due to the limitation of the basic principle of Monte Carlo stochastic simulation, the static and dynamic reliability analysis of piles based on Monte Carlo method will inevitably face a huge amount of calculation. Furthermore, most other approaches to calculating reliability depend on the Gaussian distribution, which inevitably reduces the accuracy because the correlation coefficient is changed in the nonlinear transformation from others to Normality of probability distributions [16]. In essence, the stochastic analysis of static and dynamic response of geotechnical engineering is far less than that of other distribution types, whether the physical quantities are assumed to be random variables or random processes with normal distribution. This is because probability density function of normal distribution is determined by its mean and variance. Compared with other probability information, especially the probability density function, it is much less difficult to obtain the mean and variance, and the probability density function is also the most essential description of the objective physical world. Meanwhile, all the aforementioned studies involving reliability analyses of laterally loaded piles were aimed only at static or quasi-static analysis of piles subjected to seismic loading. Less attention has been paid to the nonlinear time-history analysis of the dynamic reliability of laterally loaded piles.

When a pile is subjected to either a wind or wave load or seismic excitation, analyzing the response of that pile is a dynamic problem; if the loads being considered are in any way uncertainty or stochastic, it becomes a stochastic dynamic problem. A previous study used time-history analysis and first-passage theory to investigate the linear dynamic response and reliability of a large offshore structure considering the variability of wave loading and earthquake excitation [17]. Many studies have also used the pseudo-static method to assess the dynamic reliability of piles considering the uncertainty of earthquake ground motion [18]. Unfortunately, none of those attempts succeeded in capturing the coupling between randomness and nonlinearity. Furthermore, for stochastic dynamic analysis, Monte Carlo simulation seems to be the most universal method for a stochastic system of arbitrary dimension. However, the already huge amount of computation will grow explosively as the dimensions of the random system are increased [15,19-21], data dimensionality reduction is a common challenge for all stochastic uncertainty problems, and it is also a problem that this study needs to face. That is, by introducing a new sampling method of high-dimensional probability space, the computational complexity of stochastic static and dynamic analysis can be reduced.

Given the discussion above, the probability density evolution method is introduced herein [22]. It is used to assess the nonlinear static and dynamic responses of laterally loaded piles from the perspective of the probability density functions (PDF), which also provides a foundation for reliability analysis.

\section{Generalized Probability Density Evolution Equation}

A general static or dynamic system of laterally loaded piles subjected to static and earthquake loading can be expressed as

$$
U=\Gamma(\boldsymbol{\Theta}, \xi, u, t),
$$

where $\boldsymbol{\Theta}$ is a vector of random parameters (e.g., the uncertainty of the soil properties or the randomness of the earthquake ground motion) whose role is to describe all the possible sources of randomness in the aforementioned static or dynamic system, $\xi$ is a generalized time parameter representing the evolution direction of the system state, and $\boldsymbol{u}$ and $t$ are the spatial location vector of the system and its situated time parameter, respectively. 
The components of Equation (1) can be expressed as

$$
U_{r}=\Gamma_{r}(\boldsymbol{\Theta}, \xi, u, t)(r=1,2, \cdots, n),
$$

where $n$ is the dimension of the system and combines with the physical quantity $U_{r}$ which attracts us.

The switching rate of the system is the derivative of $U_{r}$ with respect to $\xi$ :

$$
\dot{U}_{r}(\xi)=\frac{\partial U_{r}}{\partial \xi}(r=1,2, \cdots, n) .
$$

Here, it is assumed that the stochastic static or dynamic system of the laterally loaded pile is probability conserving and that the stochastic factors of the system are given entirely by $\Theta$. The joint probability density of $(U(\xi), \Theta)$ is denoted as $p_{U \Theta}(u, \theta, \xi)$. It should be emphasized that if no new stochastic factors are added or removed in the process of system evolution, the probability of the stochastic system is conservative [23], namely,

$$
\frac{\mathrm{d}}{\mathrm{d} \xi} \int_{\Omega_{\xi} \times \Omega_{\Theta}} p_{U \Theta}(\boldsymbol{u}, \boldsymbol{\theta}, \xi) \mathrm{d} \boldsymbol{u} \mathrm{d} \boldsymbol{\theta}=0,
$$

where $\Omega_{\xi}$ is the value domain of $U$ at time $\xi$ and $\Omega_{\theta}$ is the value range of $\Theta$. $\xi_{0}$ is treated as the initial time of system evolution. Equation (4) can be rewritten as

$$
\begin{aligned}
\frac{\mathrm{d}}{\mathrm{d} \xi} \int_{\Omega_{\xi} \times \Omega_{\Theta}} p_{U \Theta}(\boldsymbol{u}, \boldsymbol{\theta}, \xi) \mathrm{d} \boldsymbol{u} \mathrm{d} \boldsymbol{\theta} & =\frac{\mathrm{d}}{\mathrm{d} \xi} \int_{\Omega_{\xi_{0}} \times \Omega_{\Theta}} p_{U \Theta \Theta}(\boldsymbol{u}, \boldsymbol{\theta}, \xi)|J| \mathrm{d} \boldsymbol{u} \mathrm{d} \boldsymbol{\theta} \\
& =\int_{\Omega_{\xi_{0}} \times \Omega_{\Theta}} p_{U \Theta}\left(\frac{\partial p_{U \Theta}}{\partial \xi}+\sum_{r=1}^{n} \dot{U}_{r} \frac{\partial p_{U \Theta}}{\partial u_{r}}\right)|J| \mathrm{d} \boldsymbol{u} \mathrm{d} \boldsymbol{\theta} . \\
& =\int_{\Omega_{\xi} \times \Omega_{\Theta}} p_{U \Theta}\left(\frac{\partial p_{U \Theta}}{\partial \xi}+\sum_{r=1}^{n} \dot{U}_{r} \frac{\partial p_{u \Theta}}{\partial u_{r}}\right) \mathrm{d} \boldsymbol{u} \mathrm{d} \boldsymbol{\theta}
\end{aligned}
$$

Because of the arbitrariness of $\Omega_{\xi} \times \Omega_{\Theta}$, the generalized probability density evolution equation (PDEE) can be obtained from Equations (3) and (4):

$$
\frac{\partial p_{U \Theta}(\boldsymbol{u}, \boldsymbol{\theta}, \xi)}{\partial \xi}+\sum_{r=1}^{n} \frac{\partial p_{U \Theta}(\boldsymbol{u}, \boldsymbol{\theta}, \xi)}{\partial u_{r}}=0
$$

in which all the variables are the same as those in Equations (2)-(4).

Equation (6) portrays the transmission regularity of the stochastic in general physical system; obviously, even the static or dynamic system of laterally loaded piles. Therefore, the PDF $p_{U}(\boldsymbol{u}, \xi)$ of $U(\xi)$ can be calculated by

$$
p_{U}(\boldsymbol{u}, \xi)=\int_{\Omega_{\Theta}} p_{U \Theta}(\boldsymbol{u}, \boldsymbol{\theta}, \xi) \mathrm{d} \boldsymbol{\theta} .
$$

The aforementioned process of solving for the PDF of a physical quality is known as the probability density evolution method (PDEM) [23,24]. Generally speaking, for a laterally loaded pile whose engineering design is investigated from the perspective of reliability, there is only one response (e.g., displacement or internal force), whereupon Equation (6) can be reduced to a one-dimensional (1D) partial differential equation, namely,

$$
\frac{\partial p_{U_{r} \Theta}\left(u_{r}, \boldsymbol{\theta}, \xi\right)}{\partial \xi}+\dot{U}_{r} \frac{\partial p_{U_{r} \Theta}\left(u_{r}, \boldsymbol{\theta}, \xi\right)}{\partial u_{r}}=0 .
$$

To solve the generalized PDEE, its initial conditions must be established here. For the 1D case, the initial conditions can be expressed as follows if the stochastic factor is without respect to the initial conditions of the pile:

$$
p_{U \Theta}(u, \boldsymbol{\theta}, \xi)_{\xi=0}=\delta\left(u-u_{0}\right) p_{\Theta}(\boldsymbol{\theta})
$$


where $u_{0}$ is the deterministic initial value of $U(\xi)$ at the time instant $\xi_{0}$ and $\delta(\cdot)$ is the Dirac delta function. In general, although the nonlinearity of the soil properties makes it hugely challenging to obtain a closed-form exact solution of Equation (6) or Equation (8), the PDEE can be solved numerically as follows:

(1) Conduct the probability division of the vector space $\Omega_{\Theta}$ of the stochastic parameters and then select representative sample point $\theta_{q}\left(q=1,2, \cdots, N_{\text {sel }}\right)$ in each subdivision, where $N_{\text {sel }}$ is the total number of subdomain divisions of the probability space and $p_{q}$ is the corresponding assigned probability measure of the subdivision region. By doing so, the stochastic response analysis of the laterally loaded pile can be translated into a series of deterministic calculations. The specific division technique (e.g., tangent sphere (TS) or number theory) can be chosen according to different probability spaces which combine with special stochastic parameters. This aspect is discussed in Sections 3.1 and 3.2 for static and dynamic problems, respectively;

(2) Introduce the deterministic response into Equation (8) as a generalized velocity $\dot{U}_{r}$, whereupon the PDF can be obtained by solving the PDEE by the finite-difference method.

\section{Numerical Cases}

This section may be divided by subheadings. It should provide a concise and precise description of the experimental results, their interpretation, as well as the experimental conclusions that can be drawn.

\subsection{Static Reliability}

In this section, the static reliability of a laterally loaded pile is investigated here (Figure 1). To compare the effectiveness and accuracy of the proposed method and solving techniques with those of existing research and the Monte Carlo method, a pile problem that has an exact solution is selected. Specifically, the lateral horizontal displacement of a pile under a force is introduced herein, the solution of which is known as the Hetényi equation $[25,26]$. Here, the diameter and length of the pile are $d=0.4 \mathrm{~m}$ and $L=5 \mathrm{~m}$, respectively, and subject it to a horizontal force $F$ and a moment $M$. It is assumed that these external influences as random variables with a correlation coefficient of $\rho(F, M)=0.5$. The material properties of the pile are described by an elastic modulus of $E_{p}=200 \mathrm{GPa}$ and a section moment of inertia of $I_{p}=3 \times 10^{-4} \mathrm{~m}^{4}$. The important parameter of the horizontal subgrade modulus $k_{h}$ is also selected as a random variable because of its variability in a free laterally loaded pile.

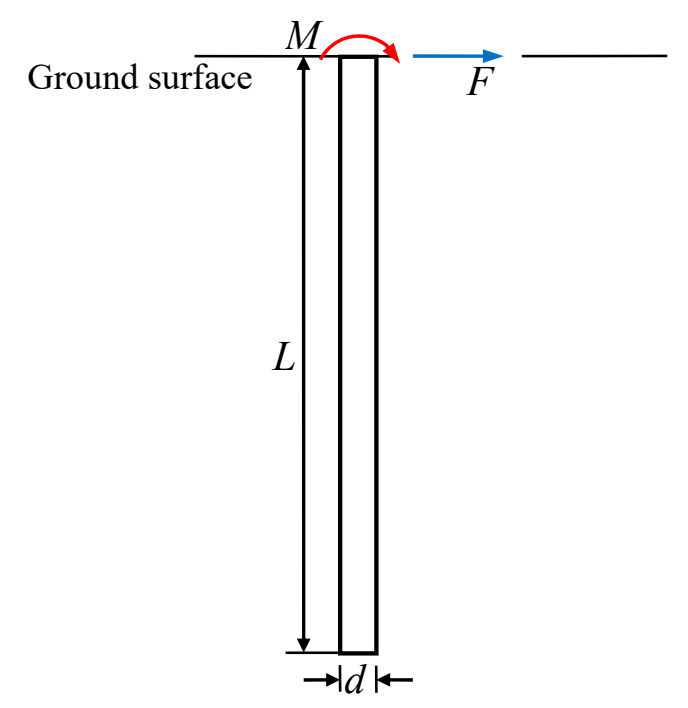

Figure 1. Laterally loaded pile. 
All the probability information regarding the random variables is listed in Table 1 . The horizontal displacement of the pile subjected to force $F$ and moment $M$ is expressed by Equations (10) and (11):

$$
\begin{aligned}
y(z) & =\frac{2 F \lambda}{k_{h} d}\left\{\frac{\sinh (\lambda L) \cos (\lambda L) \cosh [\lambda(L-z)]-\sin (\lambda L) \cosh (\lambda z) \cos [\lambda(L-z)]}{\sinh ^{2}(\lambda L)-\sin ^{2}(\lambda L)}\right\} \\
& -\frac{2 M \lambda^{2}}{k_{h} d}\left\{\frac{\sinh (\lambda L)[\cos (\lambda L) \cosh (\lambda(L-z)) \sin (\lambda z)-\sinh (\lambda(L-z)) \cos (\lambda z)]}{\sinh ^{2}(\lambda L)-\sin ^{2}(\lambda L)}\right. \\
& \left.+\frac{\sin (\lambda L)[\sinh (\lambda z) \cosh (\lambda(L-z)) \sin (\lambda z)-\cosh (\lambda z) \cos (\lambda(L-z))]}{\sinh ^{2}(\lambda L)-\sin ^{2}(\lambda L)}\right\}
\end{aligned}
$$

where $\lambda$ is a parameter expressed as

$$
\lambda=\left(\frac{k_{h} d}{4 E_{p} I_{p}}\right)^{\frac{1}{4}}
$$

Table 1. Probabilistic information on the random parameters for static reliability.

\begin{tabular}{ccccc}
\hline Parameter & Distribution & Mean & COV & $\begin{array}{c}\text { Correlation } \\
\text { Coefficient }\end{array}$ \\
\hline$F(\mathrm{kN})$ & Gumbel & 240 & 0.1 & 0.5 \\
$M(\mathrm{kN} \cdot \mathrm{m})$ & Gumbel & 80 & 0.1 & Independent \\
$k_{h}\left(\mathrm{kN} / \mathrm{m}^{3}\right)$ & Log-normal & 10,000 & 0.2 & \\
\hline
\end{tabular}

Therefore, the performance index of the pile is selected as $\left.y\right|_{z=0}=y(0)$.

According to the numerical solution process of the PDEM, the first step is to select representative sample points by dividing the probability space which combined with the random variables $F, M$, and $k_{h}$. In general, there are many random-variable sampling methods in the field of stochastic analysis. Considering sampling accuracy and efficiency, the TS point-selection technique is arguably the best one to combine with PDEM for a probability space of two or three random variables. The essence of this sampling technique is sphere packing: the probability space is represented as accurately as possible by a certain number of sampling points. Existing research suggests that there are no more than 12 equal-radius spheres tangent to a sphere in three-dimensional (3D) space. Therefore, the basic idea of TS sample points selection is that it facilitates easy construction of a coordinate representation of the centers of the TSs in 3D space. Suppose that the centers of the middle-layer spheres are located on the $z=0$ plane and that the sphere-center coordinates of the other layers in the $\mathrm{z}$ direction can be expressed as follows according to the symmetry:

$$
z_{k}=k \Delta z(k=0, \pm 1, \cdots, \pm N)
$$

where $N_{z}$ is the layer number of the TS sample technology in the $z^{\text {th }}$ layer. We assume that $\theta_{j} \in[-\eta, \eta](j=1,2,3)$ are standardized random variables and that $\eta$ is the point-selection boundary of the space of standard random variables. For instance, a reasonable range of $\eta$ is 3.2-4.0 for a standard normal distribution. Here, the value of $N_{z}$ can be taken as $\operatorname{int}\left(\frac{\eta}{\Delta z}\right)+1$, where int $(\cdot)$ represents the maximum integer that is no more than the value in the brackets and $\Delta z=\frac{2 \sqrt{6}}{3} R$, where $R$ is the radius of the TS and 1 is the number of TS layers around the sphere in the center in the $z=0$ plane.

If the sphere-center coordinates of the $z_{k}$-layer spheres are $\left(x_{i, k}, y_{i, k}, z_{i, k}\right)$, then

$$
\left\{\begin{array}{l}
x_{i, k}=x_{i, 0}+\frac{1}{2}\left[1-(-1)^{k}\right] \Delta x \\
y_{i, k}=y_{i, 0}+\frac{1}{2}\left[1-(-1)^{k}\right] \Delta y
\end{array},\left(k=0, \pm 1, \pm 2, \cdots, \pm N_{z}\right),\right.
$$


where $\Delta x=\frac{2 \sqrt{3} R \sin \psi}{3}$ and $\Delta x=\frac{2 \sqrt{3} R \cos \psi}{3}$, with $\psi=\frac{\pi}{12}$. Coordinates $\left(x_{i, 0}, y_{i, 0}, z_{k}\right)$ are those of the $z=0$ plane and can be determined by

$$
x_{i, 0}=r_{i} \cos \varphi, y_{i, 0}=r_{i} \sin \varphi,
$$

where $r_{i}$ and $\varphi$ are the radius and polar angle, respectively, in the polar coordinate system.

The joint PDF in the 3D probability space formed by the three standardized random variables $\left(\theta_{j}, j=1,2,3\right)$ is almost spherically symmetric and radially attenuated. Based on this situation, only those sampling points inside spheres of radius $\eta$ are selected. In other words, the coordinates $\left(x_{i, k}, y_{i, k}, z_{i, k}\right)$ should meet the following requirement:

$$
x_{i, k}^{2}+y_{i, k}^{2}+z_{i, k}^{2} \leq \eta^{2}
$$

Here, the coordinates are renumbered as $\left(x_{q}, y_{q}, z_{q}\right), q=1,2, \cdots, N_{s e l}$. Distribute these sampling points around the $z$ axis with fixed angle $\psi=\frac{\pi}{12}$. to make the sampling more homogeneous, therefore:

$$
\left\{\begin{array}{l}
\theta_{q 1}=x_{q} \\
\theta_{q 2}=y_{q} \cos \psi+z_{q} \sin \psi \\
\theta_{q 3}=z_{q} \cos \psi-y_{q} \sin \psi
\end{array}\right.
$$

Accordingly, the assigned probability of the representative sampling points can be calculated as

$$
P_{q}=\frac{V_{q}}{N_{s e l}} \sum_{j=1}^{N_{s e l}} p\left(\theta_{q 1, j}, \theta_{q 2, j}, \theta_{q 3, j}\right), j=1,2, \cdots, N_{s e l},
$$

where $V_{q}$ is the volume enclosed by the 12 tangent planes and $\theta_{q i, j}=\theta_{q i}+\left(\frac{r}{\eta}\right) \theta_{i, j}(i=1,2,3)$.

The representative sampling points selected above are standardized to obey a standard normal distribution on the domain $[-\eta, \eta]$. However, the target probability distributions of the random variables $F, M$, and $k_{h}$ are Gumbel and log-normal. Therefore, the sampling points should be transformed from standard normal space into the corresponding target probability space. Because doing so will change the correlation coefficient, it uses the Nataf transformation to calculate a modified correlation coefficient in light of the following approximation [16]:

$$
R_{\text {coe }}=\frac{\rho_{Y_{i} Y_{j}}}{\rho_{X_{i} X_{j}}}=1.064+(-0.069) \rho_{X_{i} X_{j}}+0.005 \rho_{X_{i} X_{j}}^{2} .
$$

where $R_{c o e}$ is the ratio of the correlation coefficients before and after transformation, $\rho_{Y_{i} Y_{j}}$ is the correlation coefficient of the target distribution, and $\rho_{X_{i} X_{j}}$ is the original correlation coefficient between $F$ and $M$, namely, $\rho_{X_{i} X_{j}}=\rho(F, M)=0.5$. Beyond that, however, the correlation coefficient can also be calculated numerically as

$$
\rho_{X_{i} X_{j}}=\int_{-\infty}^{+\infty} \int_{-\infty}^{+\infty} \frac{x_{i}-\mu_{X_{i}}}{\sigma_{X_{i}}} \frac{x_{j}-\mu_{X_{j}}}{\sigma_{X_{j}}} \varphi_{2}\left(y_{i}, y_{j} \rho_{Y_{i} Y_{j}}\right) \mathrm{d} y_{i} \mathrm{~d} y_{j}
$$

where $\mu_{X}$ and $\sigma_{X}$ are the mean and standard deviation, respectively, of random variable $X$. Herein, random variables $X_{i}$ and $X_{j}$ correspond to $F$ and $M$, respectively, with $\varphi_{2}\left(y_{i}, y_{j} \rho_{Y_{i} Y_{j}}\right)$ being the conditional cumulative distribution function (CDF).

According to Equations (18) and (19), the correlation coefficients of the target probability distributions are 0.5154 and 0.5155 , respectively. The results demonstrate that the above two categories methods are equally effective. Thus far, it has selected $N_{\text {sel }}=527$ sampling points in the 3D probability space formed by the random variables $F, M$, and $k_{h}$ according to the TS sampling technique in this 
study. By comparing the mean and variance of different sample points calculated by PDEM and Monte Carlo stochastic method, it can be found that when 527 sample points are selected, the calculation error accuracy of the random analysis can be controlled within 3\%. Therefore, 527 sample points are selected for stochastic reliability analysis. Next, a series of deterministic displacements of the laterally loaded pile can be obtained using Equations (10) and (11). Introducing the displacements into the PDEE (Equation (8)) as the generalized velocity $\dot{U}_{r}$, the PDF (Figure 2a) and CDF (Figure 2b) of the displacement of the pile top can be obtained by solving the PDEE. In Monte Carlo stochastic simulation, the reliability (CDF) is achieved by calculating the percentage of the number of samples in safe and effective state to the total number of samples by different thresholds of performance (i.e., displacement) index. The corresponding probability density function is obtained by the Kernel Density Estimation of the total sample. Different from Monte Carlo stochastic simulation, PDEM is obtained by the probability density function of performance index distribution, while the corresponding reliability $(\mathrm{CDF})$ is obtained by integrating the probability density function. The probability density function obtained by PDEM is based on the objective physical differential equation, so it has higher calculation accuracy and less calculation. The accuracy of Monte Carlo stochastic simulation is greatly affected by the calculation samples, especially the Kernel Density Estimation method, which is also affected by the selection of window function. The comparison of their accuracy can be characterized and found in Figure 2. To verify and validate the effectiveness and practicability of the PDEM and TS sampling technique, it also can calculate the PDF and CDF via kernel density estimation and Monte Carlo simulation. Herein, to compare to the previous research work [26], the number of Monte Carlo stochastic simulation also is 20,000. It should be emphasized that the accuracy and efficiency of the static reliability herein come from combining the PDEM and TS sampling technique organically. Even if with this sampling (TS) method, the Monte Carlo stochastic simulation is also impossible so effective.
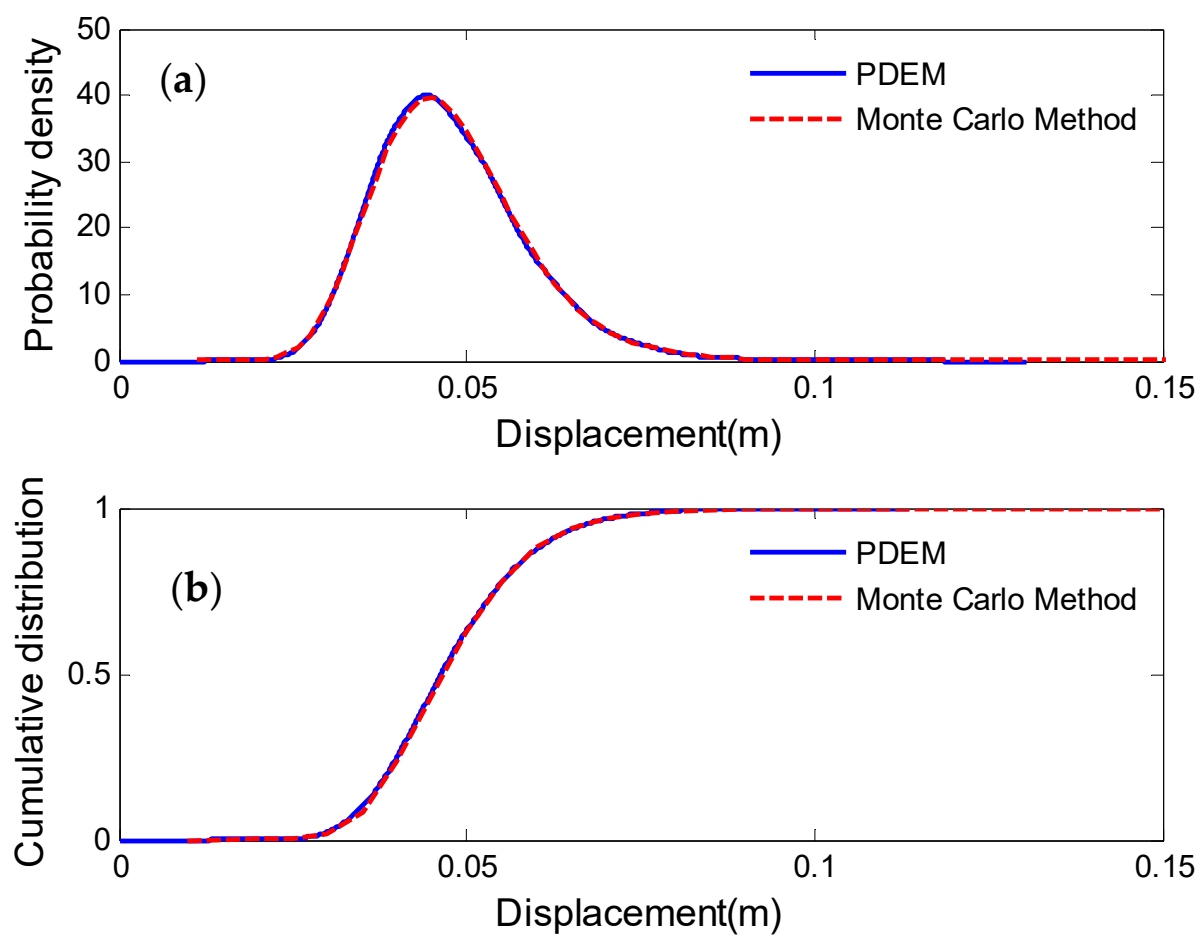

Figure 2. (a) Probability density function (PDF) of horizontal displacement of pile top. (b) Cumulative distribution function (CDF; i.e., reliability) of horizontal displacement of pile top.

\subsection{Seismic Dynamic Reliability}

In general, a laterally loaded pile subjected to seismic excitation is a strongly nonlinear dynamic system because of the nonlinear properties of concrete (i.e., the pile) and soil under earthquake dynamic 
loading. For instance, the stress-strain hysteresis curve (Figure 3) of soil under earthquake loading demonstrates that its dynamic behavior is strongly nonlinear. The deformation characteristics differ considerably under different stress states, and the nonlinearity of the limit-state equation is also a crucial distinction. Obviously, the nonlinearity means that piles are extremely sensitive to seismic excitation; the seismic dynamic responses of identical laterally loaded piles will not be exactly the same because of the stochastic nature of the earthquake ground motion. This variation is tremendous and has serious implications for seismic design and performance evaluation of laterally loaded piles. Therefore, the stochastic nature of seismic ground motion and the nonlinearity of material properties should be considered simultaneously in any system involving laterally loaded piles.

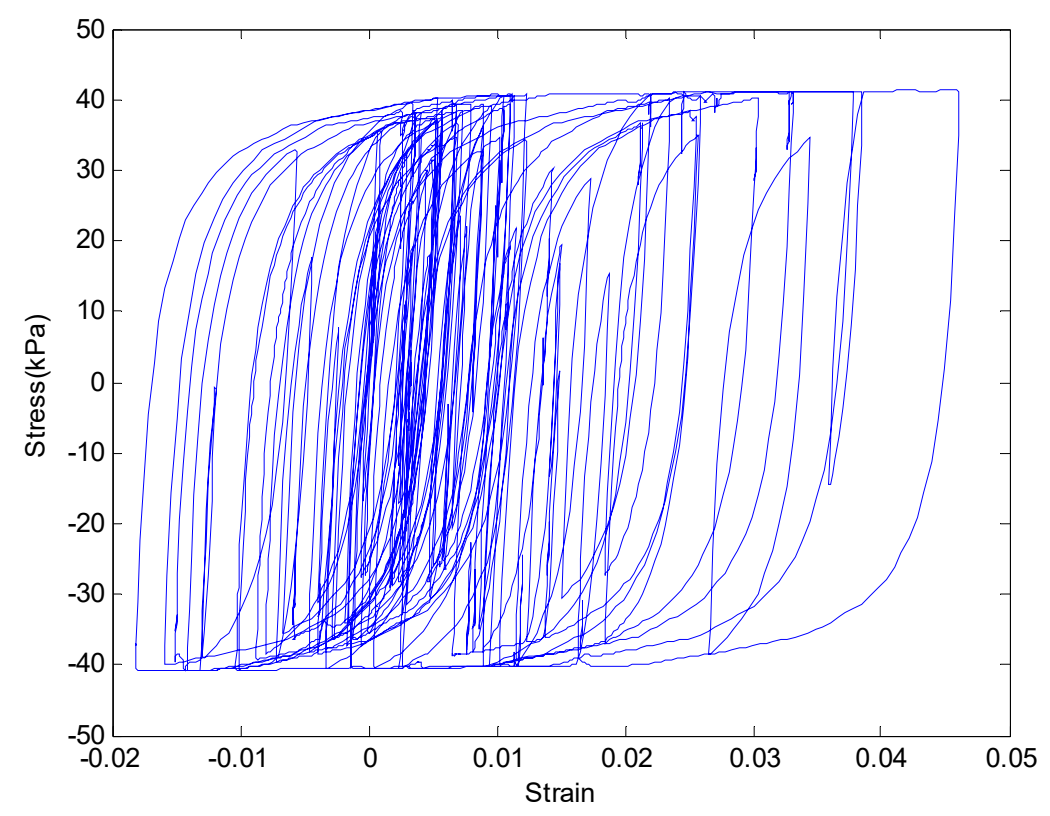

Figure 3. Stress-strain curve of soil under seismic loading.

However, the coupling between nonlinearity and randomness makes it a daunting challenge to solve for and extract the information regarding the stochastic seismic response (e.g., the PDF) of a nonlinear stochastic dynamic system. Nevertheless, the PDF of the seismic dynamic response is the foundation of any reliability calculation [27,28]. Generally speaking, only the PDF of a seismic response that obeys a Gaussian distribution can be defined by two parameters (mean and variance). Unfortunately, for most dynamic responses of a laterally loaded pile, the PDF is either not known a priori or is not Gaussian. In particular, even if the random parameters of the material properties and excitations are normally distributed, the response of the system might not have the same probability distribution as that of the initial stochastic source because of the nonlinear evolution of the system. Moreover, the PDF cannot be so easily available just only depend on the mean and variance. Hence, when analyzing the reliability of a system involving laterally loaded piles either analytically or numerically, it is essential to have access to the PDF. Here, this subsection investigates the stochastic dynamic responses of pile subjected to seismic excitation with random peak ground acceleration (PGA) and stochastic earthquake ground motion, respectively.

\subsubsection{The Stochastic Dynamic Reliability of Pile under Seismic Excitation with Random PGA}

In this section, we seek the PDF of a laterally loaded pile subjected to stochastic seismic excitation with a peak ground acceleration (PGA) given by a normally distributed random variable. 
According to the theory of stochastic dynamics, the random seismic system of a laterally loaded pile can be expressed by the following stochastic differential equation:

$$
\frac{\mathrm{d}}{\mathrm{d} t} X_{j}(t)=f_{j}[\boldsymbol{X}(t), t]+g_{j}[\boldsymbol{X}(t), t] \alpha \vartheta(t),
$$

where $\boldsymbol{X}(t)=\left[X_{1}(t), X_{2}(t), \cdots, X_{n}(t)\right]^{T}$ is the seismic response vector of the pile, $f_{j}$ and $g_{j}$ are functions representing the system properties (e.g., nonlinearity), the dynamic stress-strain curve of the soil under the deterministic seismic-acceleration time history is shown in Figure 3, which indicates that the soil goes into nonlinear state. Therefore, for nonlinear stochastic seismic dynamic response analysis, the coupling of randomness and nonlinearity is inevitable, which is also one of the difficulties in nonlinear dynamic reliability analysis. This also demonstrates that a system involving a laterally loaded pile subjected to earthquake excitation is strongly nonlinear. $\alpha$ is a random variable describing the variability of the PGA, and $\vartheta(t)$ is the time history of the deterministic seismic acceleration. Herein, it selects the strong-motion record (Figure 4) from the $1940 \mathrm{El} \mathrm{Centro} \mathrm{earthquake} \mathrm{as} \mathrm{the} \mathrm{input} \mathrm{excitation}$ and modify its PGA by the standard normal random variable $\alpha$. It is a well-known seismic record and is often selected for nonlinear dynamic response analysis. It can be found from the response spectrum (Figure 4) that the energy is mainly distributed in the low frequency part of short period. Based on the Increment Dynamic Analysis (IDA) theory, the El Centro acceleration record is selected as the input excitation for the nonlinear dynamic response analysis of pile foundation.
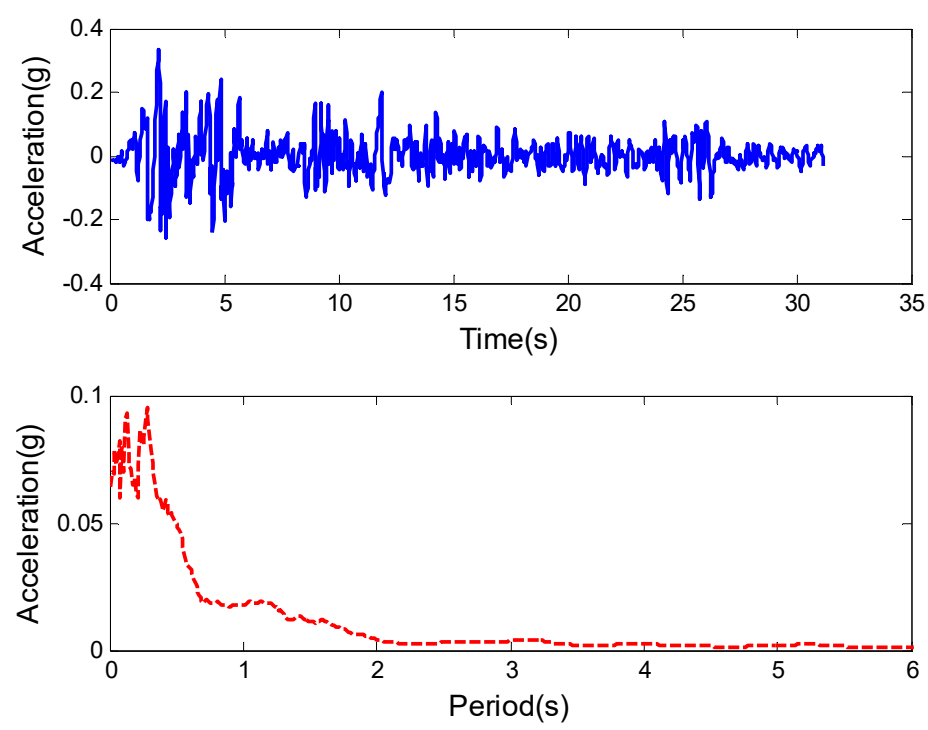

Figure 4. Seismic record (upper) and spectrum (lower) of the 1940 El Centro earthquake (NS).

Suppose that $Z(t)$ (e.g., displacement, acceleration, moment or shear force) is the important response for the seismic design of a laterally loaded pile. The PDEE can be written as

$$
\frac{\partial p_{Z}\left(z, \alpha_{q}, t\right)}{\partial t}+\dot{Z} \frac{\partial p_{Z}\left(z, \alpha_{q}, t\right)}{\partial z}=0,
$$

where $\alpha_{q}\left(q=1,2, \cdots, N_{s e l}\right)$ are the discrete representative sampling points selected in the probability space. In this section, $\alpha$ is treated as being related to a standard normal distribution and it selects roughly one thousand points (i.e., $N_{s e l}=1000$ ). By doing so, the stochastic seismic dynamic response analysis of the pile is translated into a series of deterministic dynamic time-history analyses that we conduct by FE analysis in OpenSees. The FE model is shown in Figure 5, where the pile which made by concrete and steel is modeled by dispBeamColumn elements with concrete 01 and hardening constitutive, and the soil is modeled by quad elements with $n D$ Material constitutive. The corresponding parameters 
of each constitutive are listed in Table 2, where $f_{c}$ is the concrete compressive strength, $\varepsilon_{c}$ is the concrete strain at maximum strength, $f_{c, u}$ is the concrete crushing strength, $\varepsilon_{u}$ is the concrete strain at crushing strength, $E$ is the elastic modulus, $\sigma_{s}$ is the yield stress, $H_{i}$ and $H_{k}$ are the isotropic and kinematic hardening moduli, respectively, $\rho_{0}$ is the soil mass density, $G$ is the low-strain shear modulus, and $K$ is the bulk modulus. The soil-pile interface is very important for the dynamic history analysis. The interfacial shear slip between pile and soil, diameter of pile and non-tensile effect of soil should be considered in principle. A simplified method is adopted in this paper, namely, the translational movement of nodes of soil and pile at the same place is bonded together. The equal displacement boundary conditions are used for the left and right two boundaries to keep them synchronized, which can stimulate the simplified shear boundary, the belief that the dynamic behavior of soil is simple shear movement.

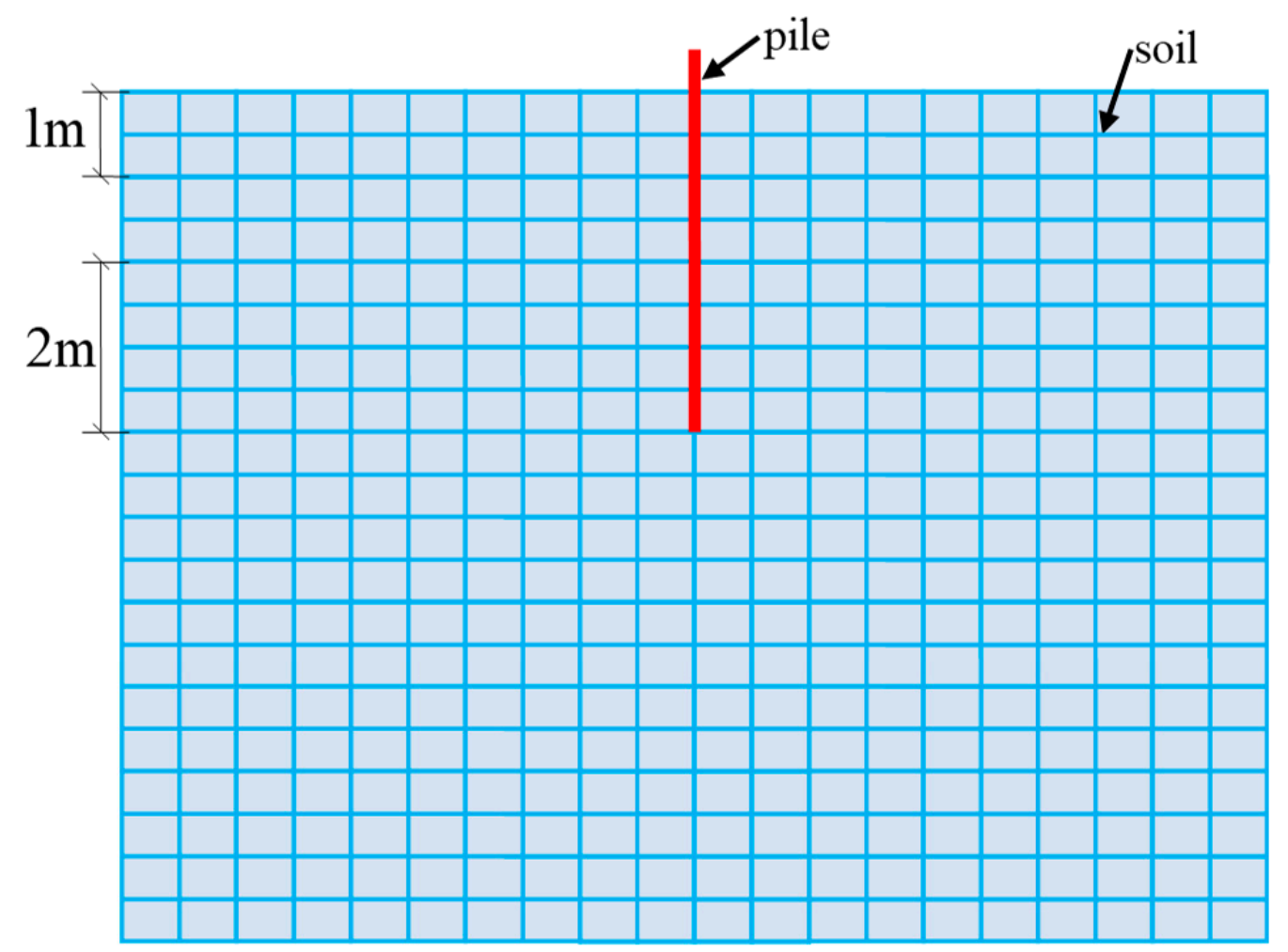

Figure 5. Finite-element (FE) model of laterally loaded pile.

Table 2. Parametric properties of the constitutive.

\begin{tabular}{cccccc}
\hline \multicolumn{2}{c}{ Concrete01 } & \multicolumn{2}{c}{ Harding } & \multicolumn{2}{c}{ nDMaterial } \\
\hline$f_{\mathcal{c}}(\mathrm{kPa})$ & -34485.6 & $E(\mathrm{kPa})$ & $2.0 \times 10^{8}$ & $\rho\left(\mathrm{kg} / \mathrm{m}^{3}\right)$ & $2.0 \times 10^{3}$ \\
$\varepsilon_{c}$ & -0.004 & $\sigma_{s}(\mathrm{kPa})$ & $248,200.0$ & $G(\mathrm{kPa})$ & 54,550 \\
$f_{c, u}(\mathrm{kPa})$ & -20691.4 & $H_{i}(\mathrm{kPa})$ & 0.0 & $K(\mathrm{kPa})$ & $1.6 \times 10^{5}$ \\
$\varepsilon_{u}$ & -0.014 & $H_{k}(\mathrm{kPa})$ & $1.6129 \times 10^{6}$ & - & - \\
\hline
\end{tabular}

For a series of deterministic nonlinear seismic dynamic time-history analyses by OpenSees (PDEM: 1000 times; Monte Carlo: 20,000 times), the nonlinear seismic dynamic response set of laterally loaded pile is achieved and introduced into the PDEE as the generalized velocity and Monte Carlo stochastic simulation to obtain the abundant probability information (e.g., PDF, mean and variance) of the stochastic dynamic system. The specific solution flowchart of PDEE is shown in Figure 6 as follows. 


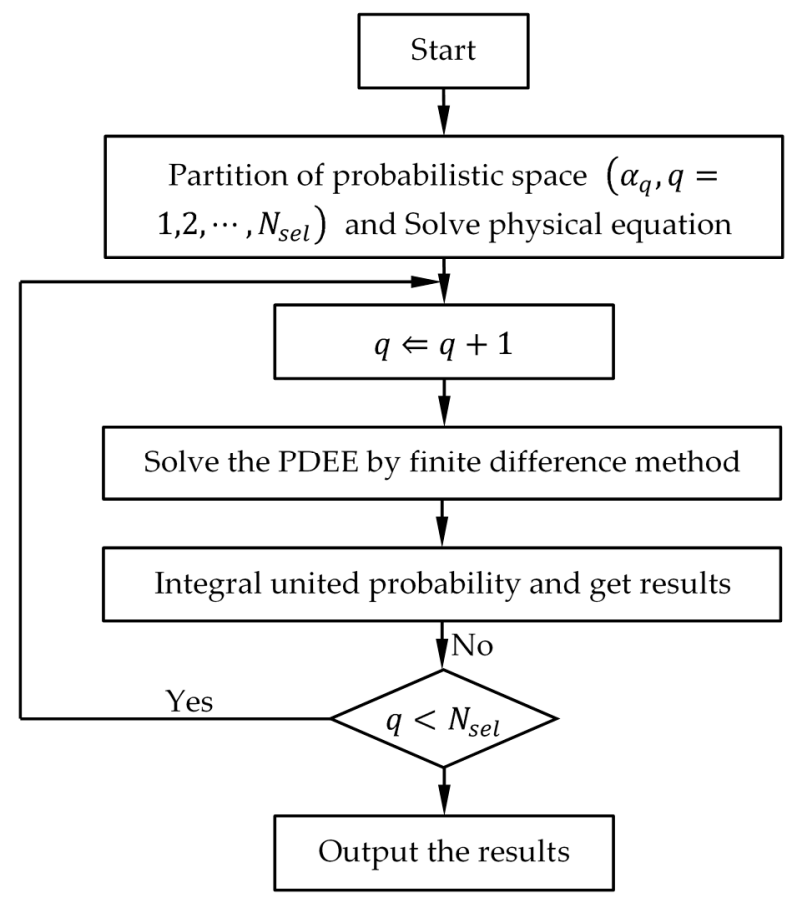

Figure 6. Computational process of PDEM.

To verify the efficiency and accuracy of the PDEM for a stochastic dynamic system, it also conducted 20,000 trials of Monte Carlo stochastic simulation. Figures 7 and 8 demonstrate the high accuracy of PDEM from the perspectives of the second-order statistics and the PDF, respectively, of the pile-top displacement.
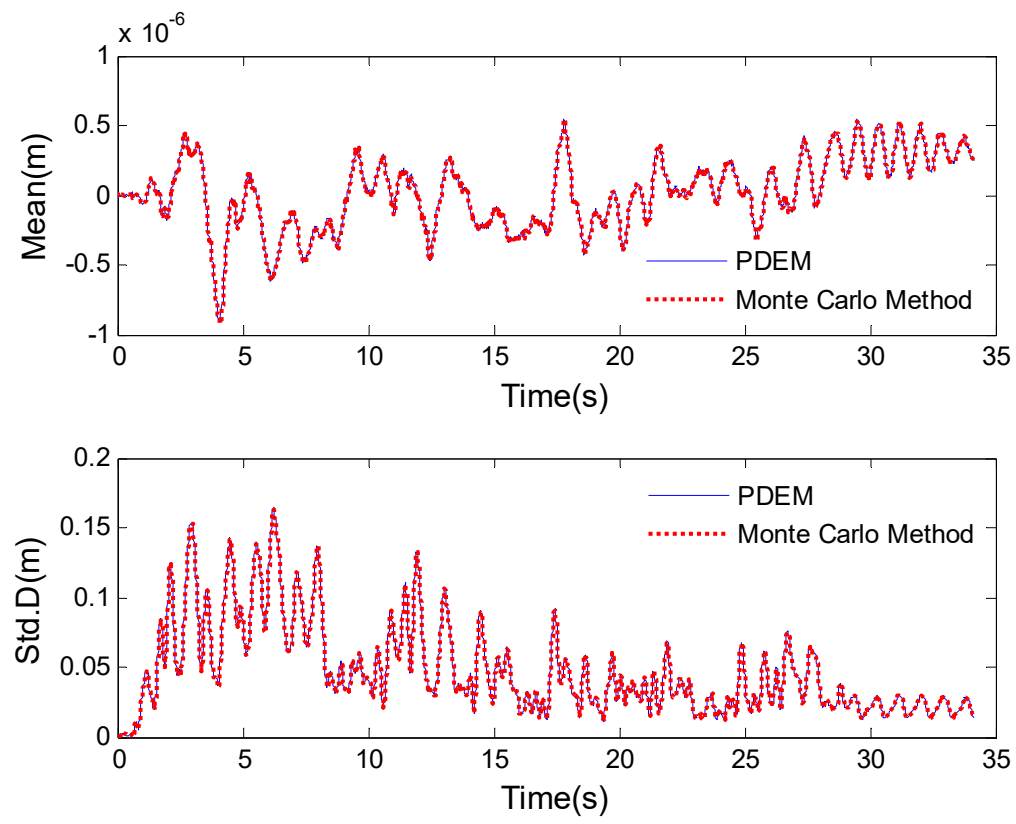

Figure 7. Mean and variance time histories of pile displacement. 


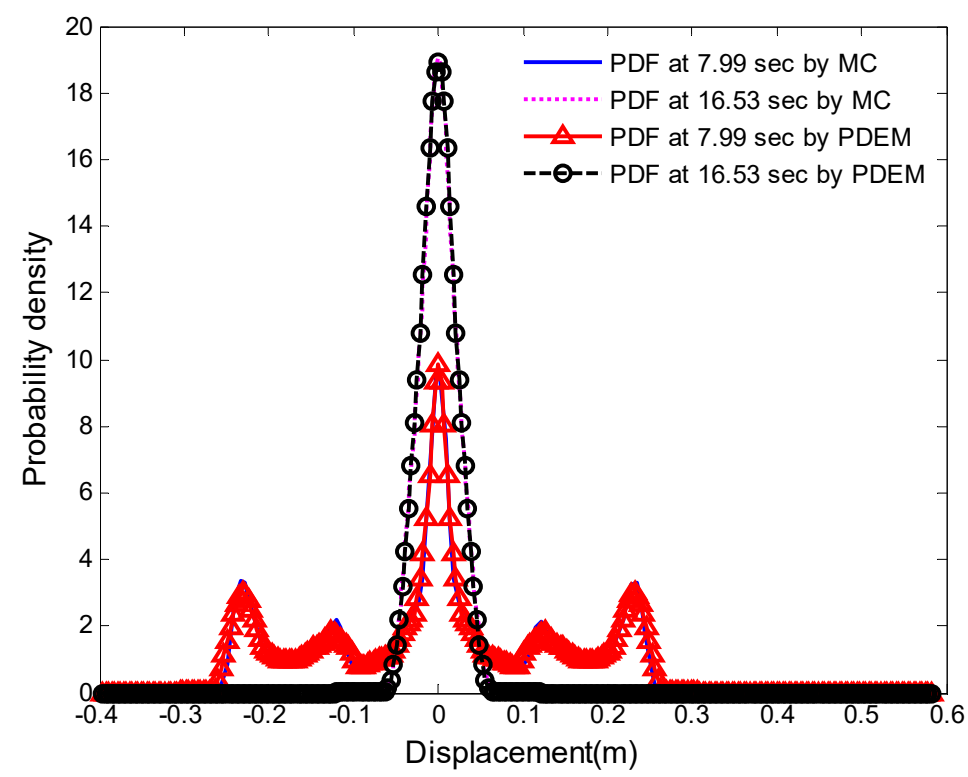

Figure 8. Comparing between probability density evolution method (PDEM) and Monte Carlo of PDFs of displacement at different times.

The gap of several orders of magnitude between the mean and standard deviation of the displacement indicates the huge variability of the seismic dynamic displacement. This characteristic is also verified by the stochastic temporal fluctuations of the PDF of the pile-top displacement.

Meanwhile, the PDF evolution surface (Figure 9) of the displacement can also be obtained by the PDEM. Figure 9 shows that the PDF evolves as the water stream and rolling hills. It also reflects the fact that the seismic dynamic displacement of the pile fluctuates with time. This is because the statement is controlled by the nonlinear properties of the system, and the evolution of probability information is transmitted by a series of samples. More importantly, the evolution driven by the coupling between nonlinearity and randomness causes the probability distribution of the displacement time history to deviate from the original distribution of the excitation. In other words, because of nonlinearity, the response to a normally distributed stochastic excitation may not be one with a Gaussian distribution.

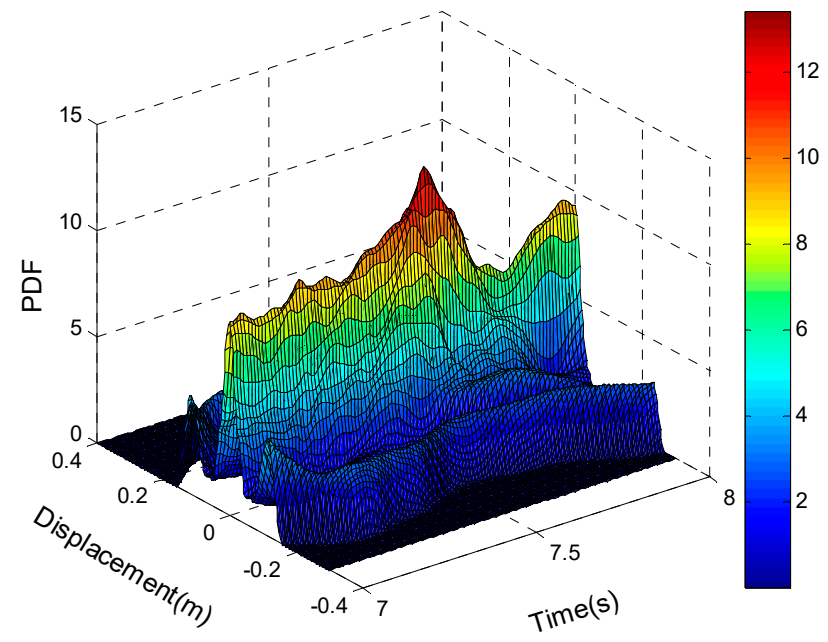

Figure 9. PDFs evolution surface of displacement.

Finally, the dynamic reliability (Figure 10) of the laterally loaded pile subjected to seismic excitation is obtained using both the PDEM and Monte Carlo stochastic simulations. It can be seen from the figure that different performance indicators correspond to different safety assurance rates, that is, the so-called 
seismic dynamic reliability of pile foundation. The validity and high accuracy of PDEM method are verified by Monte Carlo method from the aspect of seismic dynamic reliability. Furthermore, to verify the efficiency of the PDEM, the computation times for static and dynamic reliability assessments are compared in Table 3 for the PDEM and Monte Carlo simulation. Table 3 demonstrates that the PDEM is significantly more computationally efficiency compared with the classic Monte Carlo stochastic simulation. It should be noted that the Monte Carlo simulation method used in this paper is simple and plain without any other sampling techniques such as Latin hypercube. Here, the effect of different numbers $(5000,10,000$, and 20,000) of simulations on results is compared with that of PDEM by which it can find the good comparison is observed when the simulation number of Monte Carlo is 20,000.

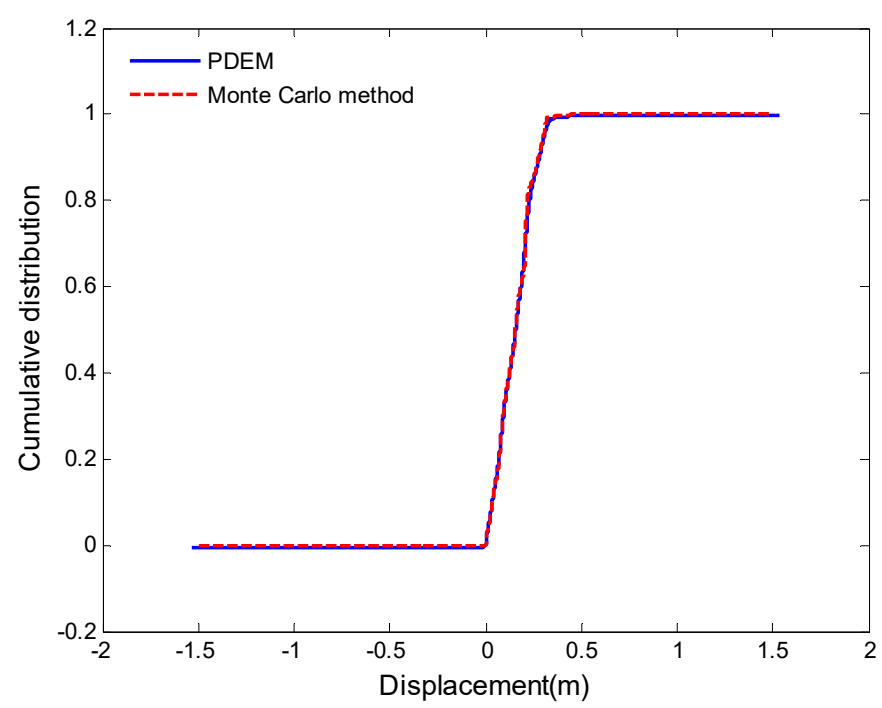

Figure 10. CDF of displacement of laterally loaded pile subjected to seismic excitation with random peak ground acceleration (PGA).

Table 3. Comparison of computation amounts and times between PDEM and Monte Carlo simulation.

\begin{tabular}{cccc}
\hline \multirow{2}{*}{ Analysis Type } & PDEM & Monte Carlo Simulation & \multirow{2}{*}{ Ratio } \\
\cline { 2 - 3 } & Samples/Time (h) & Samples/Time (h) & \\
\hline Static & 527 & 20,000 & 38 \\
Dynamic & $1000 / 5.6 \mathrm{~h}$ & $20,000 / 166.67 \mathrm{~h}$ & 20 \\
\hline
\end{tabular}

\subsubsection{The Stochastic Dynamic Reliability of Pile Subjected to Stochastic Seismic Ground Motion}

In the previous section, we investigated and discussed the stochastic seismic dynamic response and dynamic reliability of pile under the seismic action with random PGA. However, for practical engineering application, the future earthquake in the engineering site of the pile cannot be accurately predicted. Moreover, the input seismic excitation may not purely have the randomness in PGA. It should have the stochastic characteristics in both intensity and frequency. Therefore, in this section, we will continue to discuss the stochastic seismic dynamic reliability of pile subjected to stochastic seismic ground motion. Here, the stochastic dynamic difference equation Equation (20) can be modified as follows:

$$
\frac{\mathrm{d}}{\mathrm{d} t} X_{j}(t)=f_{j}[\mathbf{X}(t), t]+g_{j}[\mathbf{X}(t), t] \ddot{U}_{g}(\boldsymbol{\Theta}, t),
$$

where $\ddot{U}_{g}(\boldsymbol{\Theta}, t)$ is the stochastic seismic excitation and $\boldsymbol{\Theta}$ is the random vector which describes the randomness in intensity and frequency. The detailed generation methodology of seismic ground motion time history samples can be found in the relevant references $[29,30]$. For the stochastic seismic dynamic analysis, there are 254 deterministic dynamic time-history analyses in OpenSees with the FE model in Figure 5, and the typical acceleration time history samples are shown in Figure 11. 
It should be noted that the time histories set of the ground motion acceleration sample is the intensity frequency non-stationary random ground motion model of the same set system with corresponding given probability. It is determined based on the spectral representation of the corresponding site power spectral density function and random function. Its validity and rationality have been verified in a series of seismic dynamic evaluation of geotechnical engineering [30]. Moreover, the acceleration sample time histories in Figure 11 are only 3 out of 254 sample histories in the same set. Similarly, it also can obtain the one-dimensional PDEE Equation (8) of the key response quantity which impacts the seismic design. The CDF of displacement of laterally loaded pile subjected to stochastic seismic ground motion is shown in Figure 12.
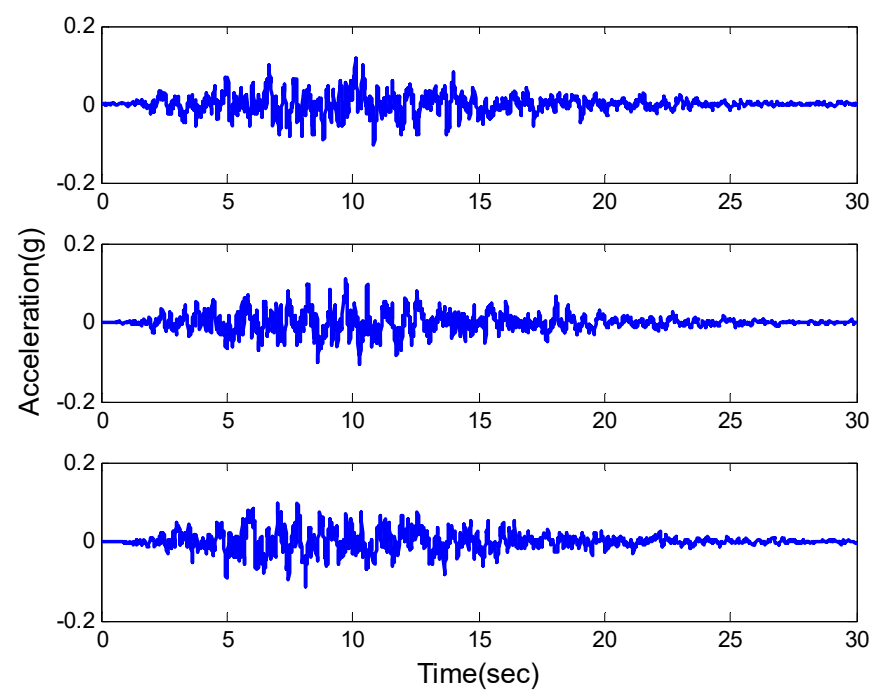

Figure 11. Typical time history samples of the seismic excitation input.

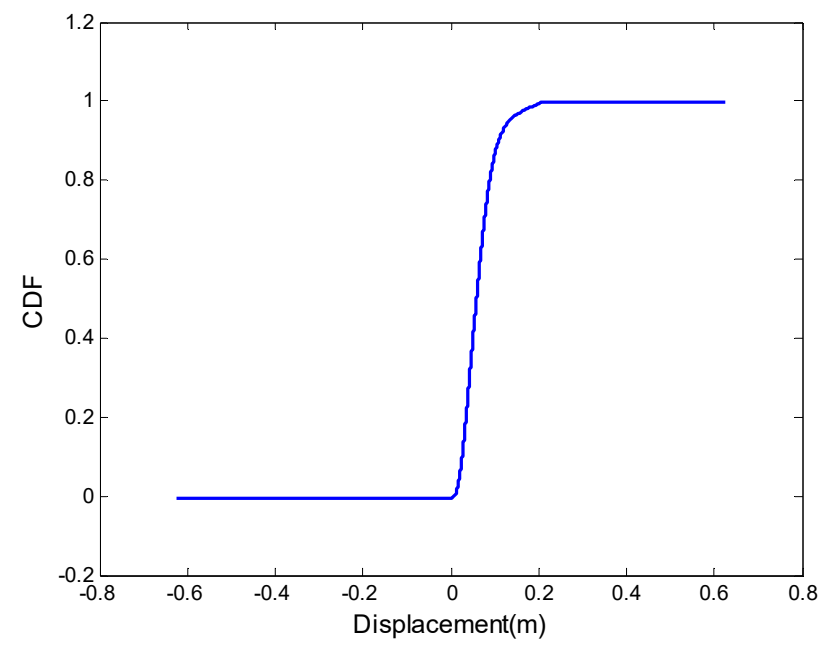

Figure 12. CDF of displacement of laterally loaded pile subjected to stochastic seismic ground motion.

\section{Conclusions}

In this paper, the static and dynamic (seismic) reliabilities of a laterally loaded pile are evaluated in light of the stochastic dynamics and a series of deterministic analyses. The innovation of this study is to introduce a new stochastic analysis method and high-dimensional spatial data reduction technology for obtaining the probability density function of multivariable stochastic static and seismic dynamic stochastic systems. The TS sampling technique is introduced to reduce the necessary sample numbers and calculation quantity. By combining TS sampling with the PDEM, the static reliability is obtained by integrating the PDF. The dynamic reliability is analyzed based on the PDEM and a series 
of deterministic time-history analyses using the FE technique. From comparisons with Monte Carlo stochastic simulation, the efficiency and accuracy of PDEM is demonstrated. The PDEM is clearly more efficient in assessing static and dynamic responses and reliability of a laterally loaded pile; when analyzing the static and dynamic reliabilities of a pile, it is roughly 38 and 20 times more efficient, respectively, than Monte Carlo stochastic simulation.

The results from the stochastic dynamic system of a laterally loaded pile subjected to seismic excitation show that the probability information is transported strongly by the nonlinear state evolution. They also indicate that the probability information of the seismic dynamic response of a pile cannot be easily determined from the excitation distribution. The assumption applied in traditional reliability analysis, namely, that the response and excitation have the same similar distribution, should be reconsidered.

Different from the classical traditional reliability analysis, especially the random analysis based on Monte Carlo stochastic simulation, the main feature of this study is to obtain the probability density function of nonlinear static and dynamic pile foundation system. Combining with the new high-dimensional probability space dimension reduction sampling technology such as TS sampling, the calculation amount of stochastic analysis, especially the nonlinear seismic dynamic reliability analysis, can be effectively reduced.

Author Contributions: M.X. and Y.H. are responsible for the preparation of this study, M.X. is responsible for performance of all the computations, the collection of data, and all the passages in this manuscript, while M.X. and Y.H. revised the paper. This study was supervised by Y.H. The results were discussed and the conclusions drafted jointly by all authors. All authors have read and agreed to the published version of the manuscript.

Funding: This research was funded by the National Natural Science Fund of China, grant number 41902274 and the National Key R\&D Program of China, grant number 2017YFC1501304.

Conflicts of Interest: The authors declare no conflict of interest.

\section{References}

1. Vogt, N.; Vogt, S.; Kellner, C. Buckling of slender piles in soft soils. Bautechnik 2009, 86, 98-112. [CrossRef]

2. Yang, J.; Ye, J.Q. Dynamic elastic local buckling of piles under impact loads. Struct. Eng. Mech. 2002, 13, 543-556. [CrossRef]

3. Liang, F.; Li, Y.; Li, L.; Wang, J. Analytical solution for laterally loaded long piles based on Fourier-Laplace integral. Appl. Math. Model. 2004, 38, 5198-5216. [CrossRef]

4. Bolton, M.D. An alternative mechanism of pile failure in liquefiable deposits during earthquakes. Geotechnique 2004, 54, 203-213.

5. Mehta, A.; Pachpor, P.D. Behavior of laterally loaded pile. Int. J. Eng. Sci. 2010, 2, 77-86.

6. Han, F.; Salgado, R.; Prezzi, M. Nonlinear analyses of laterally loaded piles-A semi-analytical approach. Comput. Geotech. 2015, 70, 116-129. [CrossRef]

7. Zhang, J.; Zhang, L.M.; Tang, W.H. Reliability based design of pile foundations considering both parameter and model uncertainties. J. Geoeng. 2009, 4, 119-127.

8. Barakat, S.A.; Malkawi, A.I.H.; Ra'ed, H.T. Reliability-based optimization of laterally loaded piles. Struct. Saf. 1999, 21, 45-64. [CrossRef]

9. Tandjiria, V.; Teh, C.I.; Low, B.K. Reliability analysis of laterally loaded piles using response surface methods. Struct. Saf. 2000, 22, 335-355. [CrossRef]

10. Kim, S.B.; Yoon, G.L.; Yi, J.H.; Lee, J.H. Reliability analysis of laterally loaded piles for an offshore wind turbine support structure using response surface methodology. Wind Struct. 2015, 21, 597-607. [CrossRef]

11. Haldar, S.; Babu, G.L.S. Design of laterally loaded piles in clays based on cone penetration test data: A reliability-based approach. Geotechnique 2009, 59, 593-607. [CrossRef]

12. Low, B.K.; Tang, W.H. Efficient spreadsheet algorithm for first-order reliability method. J Eng. Mech. 2007, 133, 1378-1387. [CrossRef]

13. Rad, M.M. Reliability based analysis and optimum design of laterally loaded piles. Period. Polytech. Civ. Eng. 2017, 61, 491-497. 
14. Schuëller, G.I. A state-of-the-art report on computational stochastic mechanics. Probab. Eng. Mech. 1997, 12, 197-321. [CrossRef]

15. Engelund, S.; Rackwitz, R. A benchmark study on importance sampling techniques in structural reliability. Struct. Saf. 1993, 12, 255-276. [CrossRef]

16. Ditlevsen, O.; Madsen, H.O. Structural Reliability Methods; Wiley: New York, NY, USA, 1996; pp. 331-335.

17. Kawano, K.; Venkataramana, K. Dynamic response and reliability analysis of large offshore structures. Comput. Methods Appl. Mech. Eng. 1999, 168, 255-272. [CrossRef]

18. Unsever, Y.S.; Matsumoto, T.; Esashi, K.; Kobayashi, S. Behaviour of model pile foundations under dynamic loads in saturated sand. Bull. Earthq. Eng. 2017, 15, 1355-1373. [CrossRef]

19. Chen, J.B.; Li, J. Stochastic seismic response analysis of structures exhibiting high nonlinearity. Comput. Struct. 2010, 88, 395-412. [CrossRef]

20. Zhang, Y.; Lam, J.S.L. Reliability analysis of offshore structures within a time varying environment. Stoch. Environ. Res. Risk. Assess. 2015, 29, 1615-1636. [CrossRef]

21. Chen, Q.; Wang, C.; Juang, C.H. Probabilistic and spatial assessment of liquefaction-induced settlements through multiscale random field models. Eng. Geol. 2016, 211, 135-149. [CrossRef]

22. Li, J.; Chen, J.B. The principle of preservation of probability and the generalized density evolution equation. Struct. Saf. 2008, 30, 65-77. [CrossRef]

23. Li, J.; Chen, J.B. Probability density evolution method for dynamic response analysis of structures with uncertain parameters. Comput. Mech. 2004, 34, 400-409. [CrossRef]

24. Chen, J.B.; Li, J. Dynamic response and reliability analysis of non-linear stochastic structures. Probab. Eng. Mech. 2005, 20, 33-44. [CrossRef]

25. Hetényi, M. Beams on Elastic Foundation: Theory with Applications in the Fields of Civil and Mechanical Engineering; University of Michigan Press: Ann Arbor, MI, USA, 1952; pp. 52-55.

26. Chan, C.L.; Low, B.K. Probabilistic analysis of laterally loaded piles using response surface and neural network approaches. Comput. Geotech. 2012, 43, 101-110. [CrossRef]

27. Wang, L.; Hwang, J.H.; Juang, C.H.; Atamturktur, S. Reliability-based design of rock slopes-A new perspective on design robustness. Eng. Geol. 2013, 154, 56-63. [CrossRef]

28. Juang, C.H.; Ching, J.; Luo, Z.; Ku, C.S. New models for probability of liquefaction using standard penetration tests based on an updated database of case histories. Eng. Geol. 2012, 133, 85-93. [CrossRef]

29. Liu, Z.J.; Liu, W.; Peng, Y.B. Random function based spectral representation of stationary and non-stationary stochastic processes. Probab. Eng. Mech. 2016, 45, 115-126. [CrossRef]

30. Huang, Y.; Xiong, M. Probability density evolution method for seismic liquefaction performance analysis of earth dam. Earthq. Eng. Struct. Dyn. 2017, 46, 925-943. [CrossRef]

Publisher's Note: MDPI stays neutral with regard to jurisdictional claims in published maps and institutional affiliations.

(C) 2020 by the authors. Licensee MDPI, Basel, Switzerland. This article is an open access article distributed under the terms and conditions of the Creative Commons Attribution (CC BY) license (http://creativecommons.org/licenses/by/4.0/). 